\section{$\mathbf{m} / \mathbf{S}$}

médecine/sciences $1999 ; 15: 1075-7$

\title{
BIOLOGIE DES GLIOMES : ACQUIS ET PERSPECTIVES CLINIQUES
}

\section{Khê Hoang-Xuan Marc Sanson Jean-Yves Delattre}

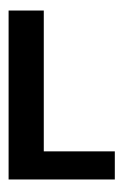

a thérapie génique par «le gène suicide " (HSVtk) a porté ces dernières années les gliomes cérébraux et plus particulièrement les glioblastomes (GBM) sur le devant de la scène de l'actualité scientifique en cancérologie [1]. Le caractère "focal ", la possibilité de cibler les cellules tumorales dans un tissu à renouvellement très lent, et le pronostic très sombre des glioblastomes (la médiane de survie est de 12 mois) expliquent que ces tumeurs aient été parmi les premières à bénéficier de ce traitement expérimental dont le développement surmédiatisé a quelque peu occulté les progrès accomplis dans le domaine biologique. Les résultats très décevants des premiers essais cliniques par rapport aux espoirs suscités [2, 3] (voir $\mathrm{m} / \mathrm{s}$ 1999, $n^{\circ} 5$, p. 591-3), sont venus nous rappeler que l'obtention à terme d'un traitement plus efficace passe par une meilleure compréhension de la biologie de ces tumeurs. Il paraît donc utile de faire le point sur le chemin parcouru cette dernière décennie dans la connaissance de la biologie des gliomes et sur les progrès à attendre dans leur prise en charge dans les prochaines années.

\section{Spécificités des gliomes}

Les tumeurs gliales présentent des caractéristiques originales qui rendent leur traitement difficile. Tout d'abord elles affectent un organe hautement fragile, le cerveau, doté d'un environnement immunologique privilégié et muni d'une "barrière hémato-cérébrale » qui le rend peu accessible aux agents administrés par voie générale. En outre, les tumeurs gliales ont une extraordinaire capacité d'infiltrer le tissu cérébral avoisinant, souvent sur de très grandes dis- tances. L'exérèse chirurgicale de la tumeur même complète en apparence n'est en réalité jamais totale et la récidive est malheureusement la règle. Autre ombre au tableau, il s'agit de tumeurs particulièrement agressives et souvent résistantes à la radiothérapie et à la chimiothérapie. Paradoxalement, ces tumeurs qui acquièrent pour la plupart un phénotype d'une extrême malignité restent en règle générale confinées au système nerveux et ne métastasent pratiquement jamais en dehors du névraxe. Les phénomènes biologiques qui sous-tendent ce phénotype particulier restent mal connus.

\section{Une classification histologique d'application difficile}

En pratique, la première difficulté, lorsque l'on parle de "gliome ", est de savoir de quelle tumeur on parle. Dans l'esprit commun, le terme de gliome est trop souvent confondu avec celui de glioblastome, qui représente certes la majorité des gliomes, mais correspond au grade de malignité le plus élevé et le plus indifférencié des tumeurs astrocytaires. Il convient de distinguer les glioblastomes des autres gliomes malins et en particulier des astrocytomes anaplasiques, des oligodendrogliomes anaplasiques et a fortiori des gliomes de bas grade. Reconnaître l'origine astrocytaire ou oligodendrocytaire de la tumeur et estimer le grade de malignité n'est pas un exercice purement académique car le pronostic, la sensibilité aux traitements et en définitive la prise en charge de ces gliomes sont différents. Là où le bât blesse, c'est qu'à l'exception relative des glioblastomes, le diagnostic histologique est parfois difficile et les critères diagnostiques demeurent très discutés. 
Cela tient à la grande hétérogénéité des gliomes qui conduit souvent à évaluer incorrectement le grade de ces tumeurs et au fait que la distinction morphologique entre un oligodendrocyte et un astrocyte tumoral est difficile en l'absence actuelle de marqueurs spécifiques fiables. A titre d'exemple, dans un essai thérapeutique international récent consacré aux astrocytomes anaplasiques, la relecture centrale des lames a montré une discordance dans trois quarts des cas par rapport au diagnostic établi par les centres locaux pourtant expérimentés. La proportion estimée des tumeurs oligodendrocytaires au sein des gliomes varie également selon l'expérience des neuropathologistes dans des proportions considérables, de $5 \%$ à $30 \%$. Certains neuropathologistes ont même remis récemment en cause l'existence de certains sous-groupes comme les astrocytomes de bas grade et anaplasiques aux profit des oligodendrogliomes [4]. Plus que jamais, la classification purement morphologique des gliomes est insuffisante et souffre de la carence de marqueurs biologiques à visée diagnostique.

\section{Du génotype au phénotype}

Pour avoir un aperçu des progrès accomplis dans le domaine biologique, faisons un bref retour en arrière et retraçons le chemin parcouru en une décennie. En 1989, le concept d'oncogène, de gène suppresseur de tumeur et de progression tumorale selon un processus «multiétapes " illustré par le modèle du cancer du côlon était déjà bien établi. Dans les gliomes, les seules altérations génétiques clairement impliquées dans la tumorigenèse de ces tumeurs étaient l'amplification de l'oncogène EGFR et les pertes du chromosome 10, bien connues des cytogénéticiens, chromosome dont on soupçonnait qu'il pouvait porter un gène suppresseur important. Grâce à l'essor de la génétique moléculaire ce sont aujourd'hui quatre gènes suppresseurs de tumeurs $(p 53$, RB, p16/CDKN2, PTEN/MMAC1 porté par le chromosome 10) et deux
CDK4) qui ont été identifiés comme étant fréquemment altérés dans les gliomes. Les fonctions des protéines codées par ces différents gènes et les mécanismes par lesquels leurs altérations participent au phénotype tumoral ont pu être précisés ces dernières années. L'intrication des différentes altérations et leurs conséquences, en particulier sur le cycle cellulaire et la transduction du signal, mais aussi sur le processus d'apoptose, d'angiogenèse et d'invasion tumorale, sont aujourd'hui mieux comprises. La confrontation des données moléculaires a révélé qu'au sein d'une même tumeur, ces altérations n'intervenaient pas de manière aléatoire mais s'associaient ou non selon une certaine cohérence [5]. Les travaux de corrélations histo-moléculaires ont permis quant à eux d'émettre des hypothèses sur l'existence de "voies moléculaires de progression tumorale » spécifiques à certains sous-groupes de gliomes et sur la chronologie de survenue de certaines altérations dans la progression tumorale. Ainsi, les mutations du gène de la p53 qui surviennent à un stade précoce semblent préférentiellement associées aux tumeurs astrocytaires caractérisées par une évolution progressive vers la malignité (astrocytomes de bas grade, astrocytomes anaplasiques, glioblastomes « secondaires ») alors que l'amplification de l'EGFR paraît associée aux glioblastomes de novo [6]. D'autres altérations comme les délétions des chromosomes $1 p$ et $19 q$ sont en revanche fréquemment associées aux tumeurs d'origine oligodendrocytaire [7]. Les gènes suppresseurs de tumeurs portés par ces régions chromosomiques restent à identifier.

En dehors de la satisfaction intellectuelle de voir valider par les données moléculaires l'existence d'entités morphologiques et évolutives reconnues de longue date, quelles applications pratiques diagnostiques et pronostiques laissent entrevoir ces découvertes? Bien qu'aucune des altérations génétiques citées ne soit suffisamment fréquente et/ou spécifique pour être utilisée à elle seule comme marqueur diagnostique en pratique courante, il ne fait guère de doute que les futures classifications des gliomes reposeront sur l'analyse conjointe des caractéristiques histologiques et du profil des altérations moléculaires des tumeurs. Un autre champ d'intérêt concerne la capacité de prévoir l'évolution tumorale des gliomes et en particulier des tumeurs de bas grade. A l'heure actuelle il est impossible de prédire à quel moment un gliome de bas grade va évoluer vers la malignité. Les délais peuvent varier de quelques mois à plus d'une dizaine d'années et l'attitude thérapeutique à adopter face à ces tumeurs demeure très controversée. L'identification d'une altération moléculaire intervenant dans le processus de transformation anaplasique des gliomes de bas grade pourrait constituer un marqueur pronostique utile. Les mutations de la p53 ont été suspectées de jouer un tel rôle dans les tumeurs astrocytaires [8], mais les études cliniques n'ont jamais pu attribuer de manière convaincante une valeur prédictive à ce marqueur. D'autres marqueurs méritent donc d'être recherchés.

\section{Vers l'intégration des données moléculaires dans la stratégie thérapeutique?}

Exceptés les progrès de la chirurgie, le traitement des glioblastomes n'a pas fondamentalement progressé depuis vingt ans. La radiothérapie a un effet limité et la place de la chimiothérapie dans le traitement initial reste controversée. Parce que certaines altérations génétiques dans les tumeurs interviennent dans les phénomènes d'apoptose et de réparation de l'ADN, elles sont susceptibles d'interférer avec la sensibilité des traitements. Leur utilisation potentielle comme facteur prédictif de réponse à la radiothérapie et aux différents agents chimiothérapiques serait utile au clinicien pour guider la stratégie thérapeutique. Dans cette optique, les oligodendrogliomes anaplasiques représentent un exemple illustratif. Il y a maintenant dix ans que Cairncross et son équipe ont montré que les oligodendrogliomes anaplasiques étaient des tumeurs chimiosensibles. Ces résultats ont été largement confirmés depuis. Récem- 
ment, la même équipe a montré qu'il existait une étroite corrélation entre la présence d'une perte des chromosomes $1 p$ et $19 q$ dans les oligodendrogliomes anaplasiques et une excellente réponse à la chimiothérapie [9]. Si ces résultats se confirment, on pourrait être bientôt en mesure de distinguer sur la base du profil moléculaire des tumeurs, les patients qui pourront bénéficier d'une chimiothérapie de ceux qui n'en tireront aucun profit. Cet exemple constitue la première étape d'un raisonnement qui devrait s'étendre aux autres types de gliomes dans le futur. Il laisse entrevoir la possibilité de traiter les patients et éventuellement de choisir les agents cytotoxiques selon des critères qui tiendront compte de la «carte d'identité moléculaire » de la tumeur.

\section{Thérapie expérimentale et essais thérapeutiques}

Outre la meilleure utilisation des moyens thérapeutiques existants, illustrée par le cas des oligendrogliomes anaplasiques, les progrès de la biologie ont permis d'identifier de nouvelles cibles thérapeutiques. Cela explique en grande partie le développement considérable de la thérapie expérimentale consacrée aux gliomes auquel nous assistons ces dernières années. Cependant, malgré des résultats expérimentaux encourageants, les traitements biologiques se sont révélés jusqu'à présent décevants chez l'homme. Les essais de traitement par anticorps monoclonaux anti-EGFR, par les inhibiteurs de la PKC (protéine-kinase $\mathrm{C}$ ), et la thérapie génique par le gène suicide HSVtk en sont malheureusement des illustrations qui posent l'éternel problème de l'adéquation des modèles expérimentaux à la pathologie humaine. Cela invite à une réflexion approfondie sur la définition de critères expérimentaux d'activité qui permettront de discerner les traitements méritant d'être testés prioritairement dans des essais cliniques. Face à un afflux prévisible de molécules «prometteuses» dans les prochaines années, il nous faudra éviter la dispersion des efforts aboutissant à des multiples essais thérapeutiques dont la qualité sera forcément médiocre, faute de puissance suffisante. Plus que jamais l'union des forces expérimentales et cliniques, la réflexion méthodologique, et la patience, loin de tout effet d'annonce intempestif seront à l'ordre du jour. Différents agents sont actuellement en cours d'évaluation chez l'homme, dirigés notamment contre l'angiogenèse (thalidomide), le processus d'invasion tumorale (marimistat), la transduction du signal (SU101) ou le métabolisme des gliomes (association lonidamide-diazépam). Il faut également citer des essais cliniques de thérapie génique à visée correctrice par transfert du gène de la p53 ou visant à stimuler l'immunité antitumorale dans les glioblastomes. Compte tenu de la complexité de la tumorigenèse des gliomes, il ne faut pas s'attendre à ce que l'inhibition d'un processus ou la correction d'une seule anomalie suffise à faire régresser de façon durable la tumeur. Les progrès viendront probablement d'un traitement intéressant plusieurs cibles à la fois et pouvant être combiné aux traitements conventionnels dont on sait qu'ils peuvent gagner à être associés de façon synergistique avec les nouvelles thérapeutiques [10]

\section{RÉFÉRENCES}

1. Culver KW, Ram Z, Wallbridge, et al. In vivo gene transfer with retroviral vector producer cell for treatment of experimental brain tumors. Science $1992 ; 256$ : 1550-2.

2. Ram Z, Culver KW, Oshiro EM, et al. Therapy of malignant brain tumors by intratumoral implantation of retroviral vector-producing cells. Nat Med 1997 ; 3 : 1354-61.

3. Klatzmann D, Valery CA, Bensimon G, et al. A phase I/II study of herpes simplex virus type 1 thymidine kinase "suicide " gene therapy for recurrent glioblastoma. Hum Gene Ther $1998 ; 9$ : 2595-604.

4. Daumas-Duport C, Varlet P, Tucker ML, Beuvon F, Cervera P, Chodkiewicz JP. Oligodendrogliomas. Part 1: Patterns of growth, histological diagnosis, clinical and imaging correlations : a study of 153 cases. INeurooncol $1997 ; 34: 37-59$.

5. Ueki K, Ono Y, Henson JW, et al. CDKN2/P16 or RB alterations occur in the majority of glioblastomas and are inversely correlated. Cancer Res 1996 ; 56 : 150-3.
6. Ohgaki H, Schauble B, Zurhausen A, Von Ammon K, Kleihues P. Genetic alterations associated with the evolution and progression of astrocytic brain tumors. Virschows Arch 1995 ; 427 : 113-8.

7. Reifenberger J, Reifenberger G, Liu L, et al. Molecular genetic analysis of oligodendroglial tumors shows preferential allelic deletions on 19q and 1p. Am J Pathol 1994; 145 : $1175-90$.

8. Sidransky M, Mikkelsen T, Schwechheimer K, Rosenblum ML, Cavenee WK, Vogelstein B. Clonal expansion of p53 mutant alleles is associated with brain tumor progression. Nature 1992; 355 : 846-7.

9. Cairncross JG, Ueki K, Zlatescu MC, et al. Specific genetic predictors of chemotherapy response and survival in patients with anaplastic oligodendrogliomas. I Natl Cancer Inst $1998 ; 90: 1473-9$

10. Badie B, Kramar MH, Lau R, et al. Adenovirus-mediated p53 gene delivery potentiates the radiation-induced growth inhibition of experimental brain tumors. $J$ Neurooncol 1998 ; 37 : 217-22.

\section{TIRÉS À PART}

K. Hoang-Xuan.

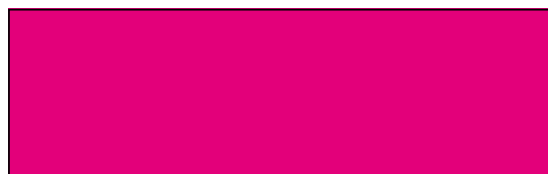

